\title{
Phytochemical Characterization and Antioxidant and Enzyme Inhibitory Activities of Different Parts of Prinsepia utilis Royle
}

\author{
Yue Zheng $\mathbb{D}^{1}{ }^{1}$ Lei Zhao $\mathbb{D}^{2}{ }^{2}$ and Junjie Yi $\mathbb{D}^{1}$ \\ ${ }^{1}$ Faculty of Food Science and Engineering, Kunming University of Science and Technology, Kunming, \\ Yunnan Province 650500, China \\ ${ }^{2}$ Beijing Engineering and Technology Research Center of Food Additives, Beijing Technology and Business University, \\ Beijing 100048, China \\ Correspondence should be addressed to Junjie Yi; junjieyi@kust.edu.cn
}

Received 28 May 2021; Accepted 25 January 2022; Published 21 February 2022

Academic Editor: Maria Concetta Strano

Copyright (c) 2022 Yue Zheng et al. This is an open access article distributed under the Creative Commons Attribution License, which permits unrestricted use, distribution, and reproduction in any medium, provided the original work is properly cited.

\begin{abstract}
The objective of this study was to evaluate the phenolic composition and antioxidant and enzyme inhibitory activities of the flowers, leaves, and stems of Prinsepia utilis Royle. In the work, their total phenol content and flavonoid content were determined. In addition, the scavenging effects of DPPH and ABTS free radicals and ferric reducing antioxidant power were measured. The results showed the flowers had the highest total phenol and flavonoid content, followed by the leaves and stems. A total of 11 phenolic substances were identified and quantified using UHPLC-ESI-HRMS/MS, of which rutin was the dominant phenolic compound in all samples. All three samples had good antioxidant activity and dose dependently inhibited the activity of $\alpha$-glucosidase, pancreatic lipase, and tyrosinase. In summary, the ethanol extracts of the flowers have the best antioxidant and enzyme inhibitory ability among three samples. The outcome could provide support for the development and utilization of P. utilis.
\end{abstract}

\section{Introduction}

Polyphenols are naturally occurring compounds that are widely distributed in different parts of plants including flowers, leaves, stems, fruits, and bark. Polyphenols play an important role in plant growth and development and protecting plants from insect damage, ultraviolet light, and wound infections [1]. In addition, they have biological functions and benefits to human health [2]. Humans ingest polyphenols mainly from fruits, vegetables, and beverages, especially tea, coffee, and red wine for good health, whereas the body cannot synthesize them by its own [3]. Therefore, it is important to adhere to daily eat a certain amount of polyphenol-rich food.

Nowadays, the incidence of chronic noncommunicable diseases (CNCDs) is increasing and the age of patients has shown a trend of getting younger, resulting from changes of diet habits, living environment, and lifestyle, for example, high-calorie food intake, industrial pollution, smoking, alcoholism, staying up late, and lack of exercise $[4,5]$.
Researchers have increasingly focused on the prevention and alleviation of the diseases through diets with little side effects. More and more research projects indicated that polyphenols have the potential to prevent or treat metabolic syndromes and many noninfectious diseases in vivo [4], so as to enhance human health. According to the previous reports, polyphenols could exhibit various biological activities including antioxidant $[6,7]$, anti-inflammatory [8], and anticancer [9], as well as the prevention of cardiovascular and Parkinson's diseases [10].

Prinsepia utilis Royle is a deciduous shrub of the Rosaceae family. It is perennial and mainly distributed in high-altitude areas, such as some areas in China and India [11]. The $P$. utilis usually grow among bushes, along depressions and roadsides, and on both sides of mountain slopes or valleys. They are cultivated as windproof and sandfixing plants that show strong adaptability to the environment. Residents in Yunnan, China, usually consume $P$. utilis as vegetables. Many parts of $P$. utilis (e.g., leaves, fruits, and roots) can be used as traditional Chinese medicine to treat 
various diseases, such as skin-related diseases, fracture, and rheumatism [12]. Previous studies have separated and identified the chemical constituents of $P$. utilis, which reported it contains hydroxybutyronitrile glucoside, diterpene glucoside, hydrocyanic acid, triterpenoids, etc [13]. However, there are few reports on the phenolic composition and biological activities of $P$. utilis flowers, leaves, and stems, which hinders the development and utilization of $P$. utilis. Therefore, the objective of this study is to compare the phenolic composition and antioxidant and enzyme (e.g., glycosidase, pancreatic lipase, and tyrosinase) inhibitory properties of different parts of P. utilis, namely flowers, leaves, and stems.

\section{Materials and Methods}

2.1. Chemical and Reagents. Acetonitrile, formic acid, Folin phenol reagent, and methanol were purchased from Darmstadt Merck, Germany. 2,2-Diphenyl-1-picrylhydrazyl radical (DPPH), 2,2'-azo-bis (3-ethylbenzothiazole-6-sulfonic acid) (ABTS), pancreatic lipase (from pig pancreas, $163 \mathrm{U} / \mathrm{mg}$, EC: 3.1.1.3), $\alpha$-glycosidase from Saccharomyces cerevisiae (type $\mathrm{I}, \geq 10$ units/mg protein), $p$-nitrobenzene $\alpha$-D-glucopyranoside (pNPG, purity $\geq 99.0 \%$ ), acarbose (purity $\geq 95 \%$ ), and orlistat (purity $\geq 97.0 \%$ ) were purchased from Sigma-Aldrich (Shanghai, China). Tyrosinase (derived from mushrooms, $500 \mathrm{U} / \mathrm{mg}$ ) was purchased from Shanghai Ruiyang (Shanghai, China). Kojic acid (purity $\geq 99.0 \%$ ) was purchased from Aladdin (Shanghai, China). Levodopa (LDOPA, purity $\geq 99.0 \%$ ) was purchased from Braunway (Beijing, China). Phenolic compound standards (purity $\geq 98.0 \%$ ) were purchased from Chengdu Bide Biotechnology Co., Ltd. (Chengdu, China). The other reagents used are of analytical grade.

2.2. Materials and Treatment. P. utilis flowers, leaves, and stems were collected from Kunming, Yunnan Province, in April 2019. After picking, they were dried and ground with a grinder (30,000 rpm; Lingdan LD-T300; Shanghai, China). A total of $100 \mathrm{~g}$ powders of the flowers, stems, and leaves were defatted with petroleum ether at a material/solvent ratio of $1: 5(\mathrm{w} / \mathrm{v})$ for five times and then extracted three times (30 min for each time) with $80 \%$ ethanol (v/v) in an ultrasonic bath. After extraction, the mixture was centrifuged at $25^{\circ} \mathrm{C}$ and $4000 \mathrm{~g}$ for $15 \mathrm{~min}$. The supernatant was collected and evaporated using a rotary evaporator (Hei-VAP, Heidolph, Germany) under vacuum at $-40^{\circ} \mathrm{C}$ to remove the organic reagent and excess water. Then, the concentrated solution was lyophilized to obtain the ethanol extracts of the flowers, stems, and leaves.

2.3. Determination of Total Phenol Content (TPC). The total phenol content of the ethanol extracts of $P$. utilis flowers, leaves, and stems was determined by using the Folin-Ciocalteu method [12]. The absorbance was measured with a microplate reader (SpectraMax M5; Molecular Device, San Jose, CA, USA) at $765 \mathrm{~nm}$, and gallic acid was used to establish the standard curve. The TPC of each sample was expressed as $\mathrm{mg}$ of GAE/g, where GAE was gallic acid equivalents.

2.4. Determination of Total Flavonoid Content (TFC). The total flavonoid content of three ethanol extracts was determined according to the methods reported in the literature [12]. The absorbance value was measured with a SpectraMax M5 microplate reader at $510 \mathrm{~nm}$, and rutin was used to establish the standard curve. The TFC of each sample was expressed as $\mathrm{mg} \mathrm{RE} / \mathrm{g}$, where RE was rutin equivalents.

2.5. Identification and Quantification of Phenolics with UHPLC-ESI-HRMS/MS. The Thermo Fisher Ultimate 3000 UHPLC system (Thermo Fisher Scientific, Bremen, Germany) with an Agilent Zorbax SB-C18 column $(2.1 \times 100 \mathrm{~mm}, 1.7 \mu \mathrm{m})$ was utilized for the qualitative and quantitative analysis of phenolic substances in the ethanol extracts of flowers, leaves, and stems from $P$. utilis. In the current study, the identified phenolic compounds were quantified by the calibration curve of the corresponding authentic standard or by the calibration curve of the standard shared a similar aglycone. The injection volume was $3 \mu \mathrm{L}$, the flow rate was $0.2 \mathrm{~mL} / \mathrm{min}$, and the column temperature was $35^{\circ} \mathrm{C}$. Acidified ultrapure water $(0.1 \%$ formic acid, phase A) and acetonitrile (phase B) were used as the mobile phases. The gradient elution condition was $0-2 \mathrm{~min}$, $5 \% \mathrm{~B} ; 2-15 \mathrm{~min}, 50 \% \mathrm{~B} ; 15-16 \mathrm{~min}, 70 \% \mathrm{~B}$. The mass spectrometry conditions were set according to the study of Sun et al. [14].

\subsection{Evaluation of Antioxidant Activity}

2.6.1. DPPH Free Radical Scavenging Experiment. The DPPH free radical scavenging ability of ethanol extracts was evaluated according to the methods of Sun et al. [14]. The absorbance of each sample was measured at $517 \mathrm{~nm}$ with a SpectraMax M5 microplate reader. The free radical scavenging capacity of DPPH is calculated by using equation (1).

DPPH free radical scavenge ratio (\%)

$$
=\frac{\left(A_{0}-A_{0}^{\prime}\right)-\left(A_{1}-A_{1}^{\prime}\right)}{A_{0}-A_{0}^{\prime}} \times 100,
$$

where $A_{1}$ represents the absorbance of sample solution and $\mathrm{DPPH}$ free radical, $A_{1}^{\prime}$ represents the absorbance of sample solution and methanol, $A_{0}$ is the absorbance of methanol and DPPH free radical, and $A_{0}^{\prime}$ is the absorbance of methanol and distilled water.

2.6.2. ABTS Free Radical Scavenging Experiment. The ABTS free radical scavenging ability of ethanol extracts was evaluated by the methods of Sun et al. [14]. The absorbance of each group was measured at $734 \mathrm{~nm}$ with a SpectraMax M5 microplate reader. The following is the calculation formula of ABTS (equation (2)): 
ABTS free radical scavenge ratio (\%)

$$
=\frac{\left(A_{0}-A_{0}^{\prime}\right)-\left(A_{1}-A_{1}^{\prime}\right)}{A_{0}-A_{0}^{\prime}} \times 100,
$$

where $A_{1}$ represents the absorbance of sample solution and ABTS free radical, $A_{1}^{\prime}$ represents the absorbance of sample solution and methanol, $A_{0}$ is the absorbance of methanol and ABTS free radical, and $A_{0}^{\prime}$ is the absorbance of methanol and distilled water.

2.6.3. Ferric Reducing Antioxidant Power (FRAP) Method. The FRAP values of three ethanol extracts were evaluated according to the reported method with some modifications [15]. Trolox $\mathrm{FeSO}_{4} \cdot 7 \mathrm{H}_{2} \mathrm{O}$ was used to prepare the calibration curve, ranging from $0.1 \mathrm{mmol} / \mathrm{L}$ to $0.5 \mathrm{mmol} / \mathrm{L}$. This curve was used to calculate the antioxidant values of each sample.

2.7. Determination of $\alpha$-Glucosidase Inhibitory Effect. The $\alpha$-glucosidase inhibitory activities of the ethanol extracts of $P$. utilis spikes, leaves, and stems were measured with the methods reported by Zhang et al. [16]. The absorbance of each reaction solution was measured at $405 \mathrm{~nm}$ with a SpectraMax M5 microplate reader. The following is the calculation formula for the $\alpha$-glucosidase inhibition (\%) (equation (3)):

$$
\begin{aligned}
& \text { glucosidase inhibition }(\%) \\
& =\frac{\left(\mathrm{OD}_{\text {control }}-\mathrm{OD}_{\text {sample }}\right)}{\mathrm{OD}_{\text {control }}} \times 100 .
\end{aligned}
$$

2.8. Determination of Pancreatic Lipase Inhibitory Effect. The pancreatic lipase inhibitory activities of the extracts of $P$. utilis flowers, leaves, and stems were measured according to the study of Cai et al. [17]. The absorbance of each reaction solution was measured at $405 \mathrm{~nm}$ with a SpectraMax M5 microplate reader. The following is the calculation formula for the pancreatic lipase inhibition (\%) (equation (4)):

$$
\begin{aligned}
& \text { Pancreatic lipase inhibition }(\%) \\
& =\frac{\left(\mathrm{OD}_{\text {control }}-\mathrm{OD}_{\text {sample }}\right)}{\mathrm{OD}_{\text {control }}} \times 100 .
\end{aligned}
$$

2.9. Determination of Tyrosinase Inhibitory Effect. The tyrosinase inhibitory activity of ethanol extracts of $P$. utilis flowers, leaves, and stems were measured according to the previously reported method with some modifications [18]. The volume of the reaction system was reduced from $1 \mathrm{~mL}$ to $200 \mu \mathrm{L}$, and the added volume of each reagent was reduced in proportion.

2.10. Statistical Analysis. All experiments were measured in triplicate, and the experimental data results were expressed as mean \pm standard deviation (SD). One-way analysis of variance and Tukey's test were used to determine the significance of difference $(p<0.05)$. All analyses were performed using Origin 8.5 software (OriginLab, Northampton, MA, USA).

\section{Results and Discussion}

3.1. Determination of Total Phenolic and Total Flavonoid Content. Table 1 shows the total phenolic and total flavonoid contents in the P. utilis flowers, leaves, and stems. Sánchez et al. [19] reported that the positive correlations between total phenol content and antioxidant capacity measured according to $\mathrm{ABTS}^{\bullet+}, \mathrm{DPPH}^{\bullet}, \mathrm{ORAC}$, and $\beta$-carotene-linoleate model system. It indicates that the total phenol content can reflect the strength of antioxidant activity of extract to a certain extent. In this study, the TPCs in the ethanol extracts of the flowers, leaves, and stems of $P$. utilis were not significantly different $(p>0.05)$, while the TPCs of dried flowers and leaves were significantly higher than that of dried stems $(p<0.05)$. The dried flowers of $P$. utilis had the highest TPC, which was 1.7 times of that of the stems.

As shown in Table 1, the TFC of $P$. utilis flower ethanol extract was significantly higher than that of leaves and stems $(p<0.05)$. The dried flowers, leaves, and stems of $P$. utilis have significant differences in TFC $(p<0.05)$, and the dried flowers had the highest TFC, followed by leaves. The TFC of the dried flowers was as about twice as that of the dried stems. According to previous reports [20], the ethyl acetate component extracted from tubers of Stachys affinis had good antioxidant activity, and the concentrations of phenols and flavonoids are positively correlated with the antioxidant activity.

\subsection{Identification and Quantitation of Phenolic Compounds.} The phenolic components of the ethanol extracts of flowers, leaves, and stems of $P$. utilis were analyzed using UHPLCESI-HRMS/MS in a negative ion mode. The ion flow chromatogram is shown in Figure 1. In addition, the relevant data such as retention time, molecular mass, molecular formula, and secondary ion fragments are shown in Table 2. The composition and content of polyphenols in the ethanol extracts of three samples were investigated by comparing the information, including secondary MS fragments with a large number of references or public mass spectrometry databases (MassBank, http://www.massbank.jp/QuickSearch.htmL). The extracts were subjected to preliminary qualitative and quantitative analysis. According to the qualitative data, appropriate standards were selected to perform further quantitative analysis on the corresponding substances. As known, the negative ion mode is usually used to detect phenolic acids and flavonoids [22]. In negative ion mode, a total of 11 compounds were analyzed including 1 phenolic acid, 10 flavonoids, and their derivatives (Table 2 and Figure 1). The retention time of flavonoids was mainly between 7 and $15 \mathrm{~min}$. In the flower extracts, the peak areas of compounds 6 and 10 were relatively high, indicating that the 
TABLE 1: Total phenolic contents (TPCs) and total flavonoid contents (TFCs) in the flower, leaf, and stem of Prinsepia utilis Royle.

\begin{tabular}{|c|c|c|c|c|c|}
\hline \multirow{2}{*}{\multicolumn{2}{|c|}{ Extraction rate (\%) }} & \multicolumn{2}{|c|}{ TPC (mg GAE/g) } & \multicolumn{2}{|c|}{ TFC (mg RE/g) } \\
\hline & & \multirow{2}{*}{$\frac{\text { DWS }}{6.40 \pm 0.07^{\mathrm{a}}}$} & \multirow{2}{*}{$\frac{\text { DWE }}{24.00 \pm 0.23^{\mathrm{a}}}$} & \multirow{2}{*}{$\frac{\text { DWS }}{31.43 \pm 0.95^{\mathrm{a}}}$} & \multirow{2}{*}{$\frac{\text { DWE }}{117.86 \pm 2.92^{\mathrm{a}}}$} \\
\hline Flower & $26.49 \pm 1.32 \%^{\mathrm{b}}$ & & & & \\
\hline Leaf & $27.59 \pm 1.37 \%^{\mathrm{a}}$ & $6.26 \pm 0.50^{\mathrm{a}}$ & $23.48 \pm 1.54^{\mathrm{a}}$ & $24.36 \pm 0.47^{\mathrm{b}}$ & $91.36 \pm 1.44^{\mathrm{b}}$ \\
\hline Stem & $17.45 \pm 0.86 \%^{\mathrm{c}}$ & $3.77 \pm 0.18^{\mathrm{b}}$ & $21.49 \pm 0.83^{\mathrm{a}}$ & $15.75 \pm 0.97^{\mathrm{C}}$ & $89.77 \pm 4.53^{\mathrm{b}}$ \\
\hline
\end{tabular}

All the values are expressed as mean $\pm \mathrm{SD}(n=3)$. Values with different superscript letters in each column indicate significant differences among samples $(p<0.05)$. DWS $=$ dry weight of sample, $\mathrm{DWE}=$ dry weight of extract, $\mathrm{GAE}=$ gallic acid equivalent, $\mathrm{RE}=$ rutin equivalents.

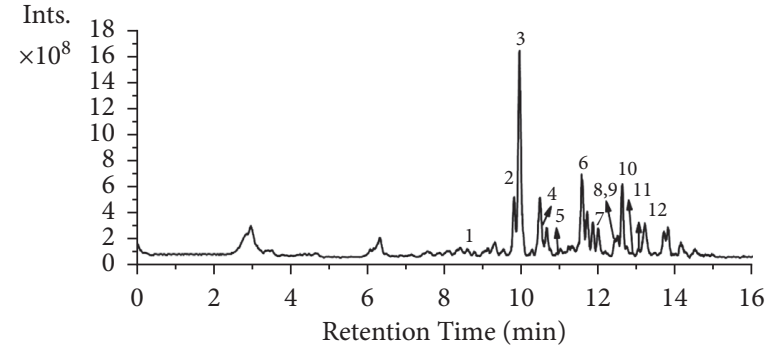

(a)

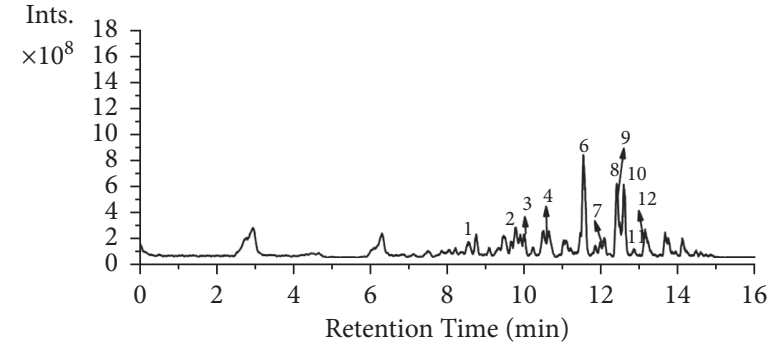

(b)

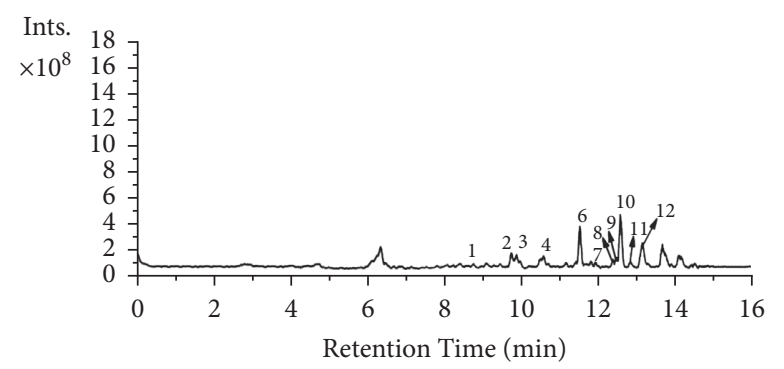

(c)

FIgUre 1: Total ion chromatograms (TICs) for the ethanol extracts of the (a) flowers, (b) leaves, and (c) stems of Prinsepia utilis Royle in negative mode. 1-12 refers to the corresponding compounds in Tables 1 and 2.

TABLE 2: Identification of major polyphenols in the flower, leaf, and stem of Prinsepia utilis Royle using the negative ion mode of UPLC-ESIHRMS/MS.

\begin{tabular}{|c|c|c|c|c|c|c|c|c|c|}
\hline $\begin{array}{l}\text { Peak } \\
\text { no. }\end{array}$ & Compounds & $\begin{array}{c}\mathrm{RT} \\
(\mathrm{min})\end{array}$ & $\begin{array}{c}{[\mathrm{M}-\mathrm{H}]^{-}} \\
(m / z)\end{array}$ & $\begin{array}{c}\text { Molecular } \\
\text { formula }\end{array}$ & MS/MS fragment ions & Reference & Flower & Leaf & Stem \\
\hline 1 & $p$-Coumaric acid & 8.57 & 163.0395 & $\mathrm{C}_{9} \mathrm{H}_{8} \mathrm{O}_{3}$ & $119.0493(100)$ & Standard & $\checkmark$ & $\checkmark$ & $\checkmark$ \\
\hline 2 & Catechin & 9.79 & 289.0726 & $\mathrm{C}_{15} \mathrm{H}_{14} \mathrm{O}_{6}$ & $\begin{array}{l}123.0443(83) \\
109.0285(100)\end{array}$ & Standard & $\checkmark$ & $\checkmark$ & $\checkmark$ \\
\hline 3 & Unknown & 9.93 & 447.1882 & $\mathrm{C}_{20} \mathrm{H}_{31} \mathrm{O}_{11}$ & $\begin{array}{l}165.0919(100) \\
220.1104(73)\end{array}$ & & $\checkmark$ & $\checkmark$ & $\checkmark$ \\
\hline 4 & Isoschaftoside & 10.53 & 563.1423 & $\mathrm{C}_{26} \mathrm{H}_{28} \mathrm{O}_{14}$ & $\begin{array}{l}563.1414(100) \\
353.0666(20)\end{array}$ & Standard & $\checkmark$ & $\checkmark$ & $\checkmark$ \\
\hline 5 & Kaempferol-3-O-glucoside & 10.95 & 447.0945 & $\mathrm{C}_{21} \mathrm{H}_{20} \mathrm{O}_{11}$ & $\begin{array}{l}284.0331(21) \\
285.0418(51)\end{array}$ & Standard & $\checkmark$ & $\checkmark$ & $\checkmark$ \\
\hline 6 & Rutin & 11.54 & 609.1474 & $\mathrm{C}_{27} \mathrm{H}_{32} \mathrm{O}_{16}$ & $\begin{array}{l}300.0283(52) \\
609.1478(100)\end{array}$ & Standard & $\checkmark$ & $\checkmark$ & $\checkmark$ \\
\hline 7 & Quercetin-3-O-glucoside & 11.97 & 463.0897 & $\mathrm{C}_{21} \mathrm{H}_{20} \mathrm{O}_{12}$ & $\begin{array}{l}300.0284(100) \\
271.0253(5)\end{array}$ & Standard & $\checkmark$ & $\checkmark$ & $\checkmark$ \\
\hline 8 & $\begin{array}{l}\text { Kaempferol-3-O- } \\
\text { rhamnosylhexose }\end{array}$ & 12.40 & 593.1535 & $\mathrm{C}_{27} \mathrm{H}_{30} \mathrm{O}_{15}$ & $\begin{array}{l}284.0334(32) \\
593.1530(64)\end{array}$ & {$[21]$} & $\checkmark$ & $\checkmark$ & $\checkmark$ \\
\hline 9 & $\begin{array}{c}\text { Quercetin 3-(6-O-acetyl-beta- } \\
\text { glucoside })\end{array}$ & 12.45 & 505.1004 & $\mathrm{C}_{23} \mathrm{H}_{22} \mathrm{O}_{13}$ & $\begin{array}{c}300.0283(100) \\
301.0347(49)\end{array}$ & $\begin{array}{l}\text { Mass } \\
\text { bank }\end{array}$ & $\checkmark$ & $\checkmark$ & $\checkmark$ \\
\hline 10 & Isorhamnetin-3-O-rutinoside & 12.60 & 623.1636 & $\mathrm{C}_{28} \mathrm{H}_{32} \mathrm{O}_{16}$ & $\begin{array}{l}315.0518(100) \\
623.1638(50)\end{array}$ & Standard & $\checkmark$ & $\checkmark$ & $\checkmark$ \\
\hline 11 & Kaempferol-3-O-hexoside & 12.87 & 447.0947 & $\mathrm{C}_{21} \mathrm{H}_{20} \mathrm{O}_{11}$ & $\begin{array}{l}285.0391(36) \\
284.0334(100)\end{array}$ & {$[21]$} & $\checkmark$ & $\checkmark$ & $\checkmark$ \\
\hline 12 & Isorhamnetin-3-O-glucoside & 13.05 & 477.1052 & $\mathrm{C}_{22} \mathrm{H}_{22} \mathrm{O}_{12}$ & $\begin{array}{c}314.0442(100) \\
477.1052(25)\end{array}$ & Standard & $\checkmark$ & $\checkmark$ & $\checkmark$ \\
\hline
\end{tabular}

$\mathrm{RT}=$ retention time, $\checkmark=$ contains the substance. 
two phenolic substances are the main compounds in the ethanol extract of $P$. utilis flowers. Compound $6\left([\mathrm{M}-\mathrm{H}]^{-}\right.$at $\mathrm{m} / z$ 609.1474) was determined as rutin by comparison with the corresponding standard. The characteristic ion fragment of compound $6(\mathrm{~m} / z=300.0283)$ was caused by the partial loss of rutinose. Compound $10\left([\mathrm{M}-\mathrm{H}]^{-}\right.$at $\mathrm{m} / z$ 623.1636) was identified as isorhamnetin-3-O-rutinoside by comparison with the standard and the presence of $\mathrm{m} / z$ 315.0518, which represent the loss of a rutinose in isorhamnetin-3-Orutinoside produces this characteristic ion. Among all the identified components, rutin (compound 6) was the predominate phenol in the leaf and stem extracts. Additionally, the peak areas of catechin, kaempferol-3-O-glucoside, and quercetin-3-O-glucoside were higher than that of the other components.

The quantitative analysis results of all phenolic components in the ethanol extracts of $P$. utilis flowers, leaves, and stems are shown in Table 3. Among them, there were 8 compounds $(1,2,4,5,6,7,10$, and 12) that can be analyzed by comparison with the corresponding standards. The rest of the phenolic substances $(8,9$, and 11) can use similar aglycone standards for their quantitation. As shown in Table 3, rutin was the dominant phenolic compound in all the extracts. However, the rutin content was different among three extracts, that the highest content was detected in flowers, followed by leaves and stems. It indicates that phenol contents and species in the ethanol extracts of $P$. utilis flowers, leaves, and stems were abundant, which may have good biological activity. Zhang et al. [16] studied the composition and biological activity of the total phenols, total flavonoids, and anthocyanin components in P. utilis fruits. A total of 20 phenolic compounds were identified, of which composition was similar to that of ethanol extracts of $P$. utilis flowers, leaves, and stems. Zhang et al. found that three main components (rutin, isorhamnetin-3-O-rutinoside, and cyanidin-3-O-glucoside) in $\mathrm{P}$. utilis fruits have a good inhibitory effect on $\alpha$-glycosidase and lipase. The ethanol extracts of the flowers, leaves, and stems of $P$. utilis also contain rutin and isorhamnetin-3-O-rutinoside.

3.3. In Vitro Antioxidant Activity. Free radicals are inevitably produced in biological systems and taken into the body from the environment. They are known to cause a variety of degenerative diseases, such as mutagenesis, carcinogenesis, cardiovascular disorders, and aging [23]. An antioxidant is a compound that inhibits free radicals by interfering with the three main steps of the free radical-mediated oxidation process, namely initiation, proliferation, and termination [24]. The balance between oxidants and antioxidants determined inside the human body is important to protect against the occurrence of many diseases [25].

Generally, plant polyphenols have been reported to show many important biological activities, which is believed to be partially or largely related to their antioxidant activity [15]. At present, some in vitro antioxidant methods (e.g. DPPH, ABTS, and FRAP) are usually used to measure the antioxidant capacity of a substance. The obtained results are used as a reference index for the antioxidant effect in vivo. In the work, DPPH, ABTS, and FRAP methods were used to evaluate the antioxidant activity of the ethanol extracts of $P$. utilis flowers, leaves, and stems.

3.3.1. DPPH Free Radical Scavenging Activity. The DPPH free radical scavenging activity of ethanol extracts from different parts of $P$. utilis is shown in Figure 2(a). In the concentration range of $20-100 \mu \mathrm{g} / \mathrm{mL}$, the DPPH free radical scavenging activity of all samples was concentration-dependent. The DPPH free radical scavenging activity of the flower extract of $P$. utilis was significantly stronger than that of the other two samples $(p<0.05)$. The $\mathrm{IC}_{50}$ values of the ethanol extracts of $P$. utilis flowers, leaves, and stems were $46.07 \pm 2.42,82.08 \pm 0.97$, and $71.46 \pm 2.85 \mu \mathrm{g} / \mathrm{mL}$, respectively. The scavenging rate of DPPH free radicals of three samples is lower than that of $\mathrm{Vc}$ (positive control, $\mathrm{IC}_{50}=3.75 \pm 0.02 \mu \mathrm{g} / \mathrm{mL}$ ). The flower extract has the strongest DPPH scavenging ability among the three components $(p<0.05)$, which may be attributed to its highest polyphenol content.

3.3.2. ABTS Free Radical Scavenging Activity. Figure 2(b) shows the ABTS free radical scavenging ability of the ethanol extract of $P$. utilis flowers, leaves, and stems. The $\mathrm{IC}_{50}$ values of the ethanol extracts of $P$. utilis flowers, leaves, and stems for scavenging ABTS free radicals were $22.07 \pm 0.94$, $27.14 \pm 0.28$, and $20.29 \pm 0.57 \mu \mathrm{g} / \mathrm{mL}$, respectively. The $\mathrm{IC}_{50}$ values of the ethanol extracts of flowers, leaves, and stems of $P$. utilis for ABTS scavenging assay were $22.07 \pm 0.94$, $27.14 \pm 0.28$, and $20.29 \pm 0.57 \mu \mathrm{g} / \mathrm{mL}$, respectively. It shows that the ethanol extract of leaves had the best scavenging effect on ABTS free radicals among all ethanol extracts. The $\mathrm{IC}_{50}$ value of $\mathrm{Vc}$ was $3.56 \pm 0.07 \mu \mathrm{g} / \mathrm{mL}$, and its ABTS scavenging effect is better than that of the P. utilis extracts. Overall, the ethanol extracts of flowers, leaves, and stems from $P$. utilis had strong free radical scavenging activity. Three extracts had different $\mathrm{IC}_{50}$ values for their DPPH and ABTS free radical scavenging activities, which may be caused by the differences in free radical species, polyphenol compositions, and contents.

3.3.3. FRAP Evaluation. FRAP is a relatively efficient and sensitive method to determine the antioxidant capacity of samples, which is widely used in food, chemical, and other fields. The antioxidant capacity of a sample evaluated using this method is expressed by the equivalent value of $\mathrm{FeSO}_{4}$. The concentration of $\mathrm{FeSO}_{4}$ was in the range of $0.1 \sim 0.5 \mathrm{mmol} / \mathrm{L}$, and the concentration has a good linear relationship with the absorbance at $593 \mathrm{~nm}$. Figure 2(c) shows the ferrous reduction capacity of the ethanol extracts of $P$. utilis. The concentration of three group samples filled in the range of $20-60 \mu \mathrm{g} / \mathrm{mL}$. The flower extract had the highest FRAP value $(p<0.05)$, followed by the leaf extract and the stem extract. For example, the ferrous reduction activity of flower extract was significantly higher than that of leaf and stem extracts $(p<0.05)$ at $60 \mu \mathrm{g} / \mathrm{mL}$. The FRAP values of flower, leaf, and stem extracts were $0.60 \pm 0.01$, 
TABle 3: Quantitation of phenolic substances in the flower, leaf, and stem of Prinsepia utilis Royle by UPLC-ESI-HRMS/MS.

\begin{tabular}{lcccc}
\hline Peak no. & Compounds & Flower $(\mu \mathrm{g} / \mathrm{g})$ & Leaf $(\mu \mathrm{g} / \mathrm{g})$ & Stem $(\mu \mathrm{g} / \mathrm{g})$ \\
\hline 1 & p-Coumaric acid & $381.60 \pm 19.08^{\mathrm{a}}$ & $265.60 \pm 13.28^{\mathrm{a}}$ & $22.85 \pm 1.14^{\mathrm{b}}$ \\
2 & Catechin & $742.60 \pm 37.13^{\mathrm{a}}$ & $448.86 \pm 22.44^{\mathrm{b}}$ & $227.99 \pm 11.40^{\mathrm{c}}$ \\
4 & Isoschaftoside & $463.58 \pm 23.18^{\mathrm{b}}$ & $352.65 \pm 17.63^{\mathrm{c}}$ & $537.65 \pm 26.88^{\mathrm{a}}$ \\
5 & Kaempferol-3-O-glucoside & $9.39 \pm 0.47^{\mathrm{a}}$ & $\mathrm{ND}$ & $\mathrm{ND}^{\mathrm{a}}$ \\
6 & Rutin & $8822.63 \pm 441.13^{\mathrm{a}}$ & $8484.19 \pm 424.21^{\mathrm{a}}$ & $1801.33 \pm 90.07^{\mathrm{b}}$ \\
7 & Quercetin-3-O-glucoside & $744.64 \pm 37.23^{\mathrm{a}}$ & $237.85 \pm 11.89^{\mathrm{b}}$ & $33.54 \pm 1.68^{\mathrm{c}}$ \\
8 & Kaempferol-3-O-rhamnosylhexose & $269.34 \pm 13.47^{\mathrm{b}}$ & $1069.80 \pm 53.49^{\mathrm{a}}$ & $29.14 \pm 1.46^{\mathrm{c}}$ \\
9 & Quercetin 3-(6-O-acetyl-beta-glucoside) & $668.61 \pm 33.43^{\mathrm{a}}$ & $46.00 \pm 2.30^{\mathrm{b}}$ & $8.08 \pm 0.40^{\mathrm{b}}$ \\
10 & Isorhamnetin-3-O-rutinoside & $1944.96 \pm 97.25^{\mathrm{a}}$ & $1108.94 \pm 55.45^{\mathrm{b}}$ & $529.00 \pm 26.45^{\mathrm{c}}$ \\
11 & Kaempferol-3-O-hexoside & $65.72 \pm 3.29^{\mathrm{a}}$ & $23.81 \pm 1.19^{\mathrm{b}}$ & $3.91 \pm 0.20^{\mathrm{c}}$ \\
12 & Isorhamnetin-3-O-glucoside & $30.34 \pm 1.52^{\mathrm{c}}$ & $57.71 \pm 2.89^{\mathrm{a}}$ & $37.60 \pm 1.88^{\mathrm{b}}$ \\
\hline
\end{tabular}

All the values are expressed as mean $\pm \mathrm{SD}(n=3)$. Values with different superscript letters in each column indicate significant differences among samples. Compounds $1,2,4,5,6,7,10$, and 12 were quantified by the calibration curve of the corresponding commercial standard; compounds 8 and 11 were quantified by the calibration curve of compound 5; and compound 9 was quantified by the calibration curve of compound 7 . The result is expressed as $\mu \mathrm{g} / \mathrm{g}$ dry matter. $\mathrm{ND}=$ not detected.

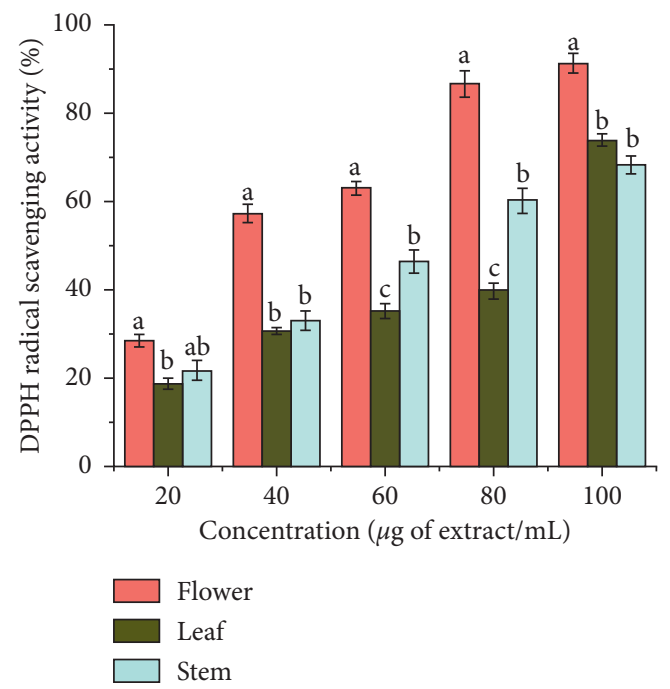

(a)
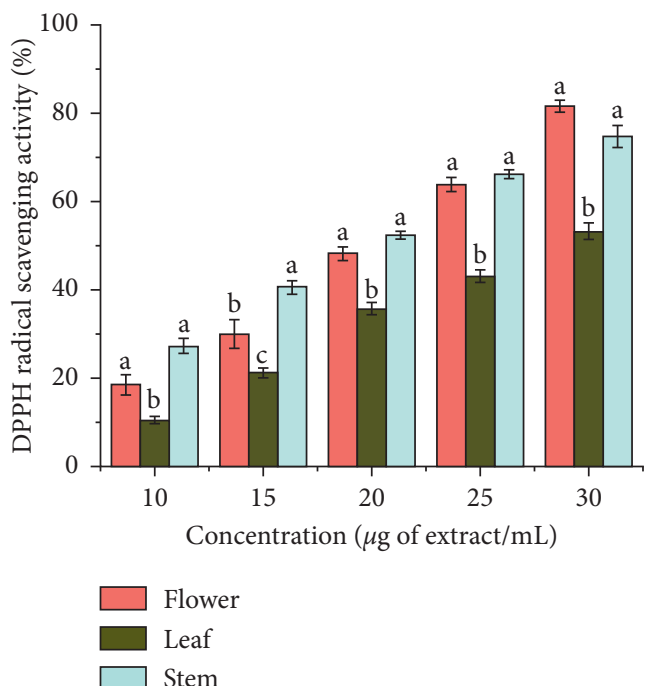

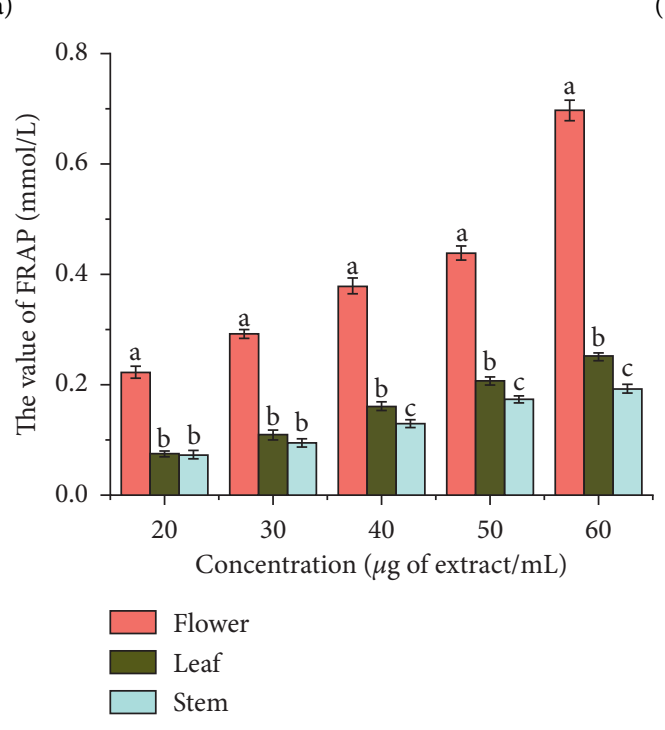

(b)

(c)

FIgURE 2: The antioxidant activities of the flower, leaf, and stem extracts of Prinsepia utilis Royle. (a) DPPH radical scavenging activity, (b) ABTS radical scavenging ability, and (c) ferric reducing antioxidant power (FRAP). All the values are expressed as mean \pm SD ( $n=3$ ). Values with different superscript letters in each column indicate significant differences among samples $(p<0.05)$. 


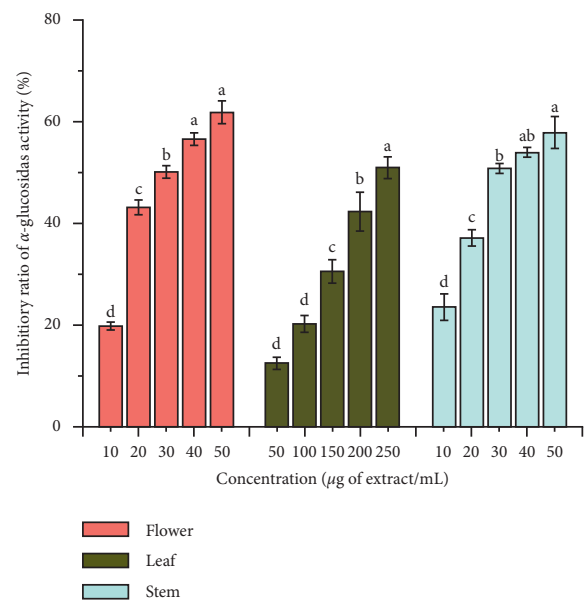

(a)

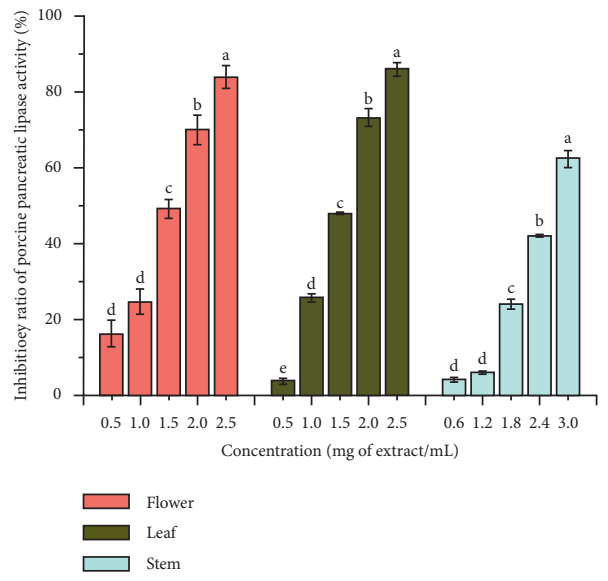

(b)

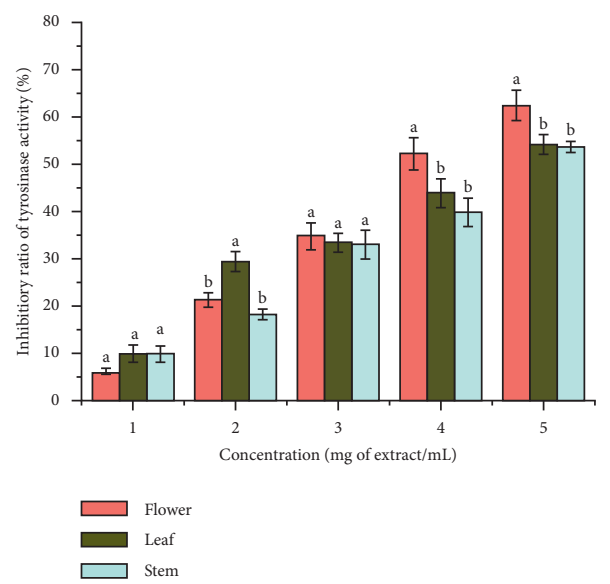

(c)

FIgURE 3: (a) $\alpha$-glucosidase inhibitory rates, (b) porcine pancreatic lipase inhibitory rates, and (c) tyrosinase inhibitory rates of the flower, leaf, and stem extracts from Prinsepia utilis Royle. All the values are expressed as mean \pm SD $(n=3)$. Values with different superscript letters in each column indicate significant differences among samples $(p<0.05)$.

$0.25 \pm 0.003$, and $0.19 \pm 0.01 \mathrm{mmol} / \mathrm{L}$, respectively. The ferrous reduction activity of flower extract was 3 times higher than that of stem extract and 2.4 times higher than that of leaf extract. In addition, it can be observed that the samples with high total phenol content have high FRAP values.

In general, the antioxidant activity of the $P$. utilis flower ethanol extract was the highest among all three samples. It may be due to the fact that the total phenol and total flavonoid contents of flower extract was higher than other samples. Huang et al. [11], Zhang et al. [26], and Zhang et al. [16] reported that $P$. utilis fruits showed a higher antioxidant capacity than flowers, leaves, and stems. Although the total phenol and total flavonoid contents in leaves are higher than those in stems, the ethanol stem extract had a stronger free radical scavenging ability than that of leaf extract. It was found that the content of isoschaftoside in the ethanol extract of stem was higher than that of leaf, but the other components of the ethanol extract of stems were lower than that of the leaf extract. Compared with the leaf extract, the better scavenging effect of stem extract against DPPH and ABTS free radicals may attribute to its higher content of isoschaftoside. A previous study reported a similar finding that the DPPH scavenging activity of ethanol extract from Okinawa Taumu (Colocasia esculenta S.) was higher than that of the water extract [27]. In addition to phenolic compounds, some other components also exhibited a good antioxidant activity, such as peptides [28]. Therefore, the total antioxidant activity of a sample was contributed from a combination of several components.

3.4. $\alpha$-Glucosidase Inhibitory Activity. Figure 3(a) shows the inhibitory effect of ethanol extracts of $P$. utilis flowers, leaves, and stems on $\alpha$-glucosidase. The $\alpha$-glucosidase inhibitory effects of three extracts were concentration-dependent. As shown in Figure 3(a), the $\mathrm{IC}_{50}$ values for the $\alpha$-glucosidase inhibitory activity of ethanol extracts from $P$. utilis spike flowers, leaves, and stems were $35.71 \pm 0.58,231.12 \pm 6.59$, and $37.29 \pm 1.59 \mu \mathrm{g} / \mathrm{mL}$, respectively. The $\mathrm{IC}_{50}$ value of the positive control acarbose was $0.16 \pm 0.04 \mu \mathrm{g} / \mathrm{mL}$, which was significantly lower than that of three extracts $(p<0.05)$. The flower extracts had the highest inhibitory effect on 
$\alpha$-glucosidase, followed by stem and leaf extracts, which may be due to the different polyphenol composition. Several studies $[29,30]$ reported that plants rich in polyphenols have good inhibitory activity on $\alpha$-glucosidase. It suggested that phenolic compounds are considered to be the main compounds that contribute to the $\alpha$-glucosidase inhibitory activity. Zhang et al. [16] have reported that the $P$. utilis fruits were rich in rutin and isorhamnetin-3-O-rutinoside, and those phenolic compounds exhibited a good inhibitory activity towards $\alpha$-glucosidase.

3.5. Pancreatic Lipase Inhibitory Activity. Figure 3(b) shows the pancreatic lipase inhibitory activity of $P$. utilis flower, leaf, and stem ethanol extracts. As shown in Figure 3(b), each extract had a good inhibitory effect on pancreatic lipase. As the concentration increased, the pancreatic lipase inhibition rates of three extracts gradually increased. The $\mathrm{IC}_{50}$ values for pancreatic lipase inhibitory activity of the ethanol extracts from $P$. utilis flowers, leaves, and stems were $1.72 \pm 0.03,1.82 \pm 0.15$, and $2.56 \pm 0.02 \mathrm{mg} / \mathrm{mL}$, respectively. It can be seen that the flower extract had the highest inhibitory activity on pancreatic lipase, followed by the leaf and stem extracts. However, orlistat, as a positive control, inhibited almost $50 \%$ of lipase activity at $40.00 \mu \mathrm{g} / \mathrm{mL}$ in the current work, indicating that orlistat may possess much stronger inhibitory effect against lipase than the three extracts. Considering the total phenol content of three samples, it was found that the sample with higher polyphenol content had better inhibitory activity on pancreatic lipase. Many studies have shown that plant food extracts rich in phenols generally have good inhibitory activity against lipases [31]. Dorota et al. reported the inhibitory effects of the crude extract of black chokeberry fruit and its ethyl acetate fraction and polyphenol-rich fraction on pancreatic lipase activity [31]. The results showed that the polyphenol-rich fraction exhibited higher antilipase activity than other components. In general, the composition and content of phenolics in the extract may play an important role in pancreatic lipase inhibitory activity.

3.6. Tyrosinase Inhibitory Activity. Figure 3(c) shows the inhibition of tyrosinase activity of ethanol extracts of $P$. utilis flowers, leaves, and stems. As the concentration increased, the inhibitory effect of each extract on tyrosinase gradually increased. The $\mathrm{IC}_{50}$ values of the P. utilis flower, leaf, and stem extracts for tyrosinase inhibitory activity were $4.11 \pm 0.11,4.33 \pm 0.04$, and $4.58 \pm 0.11 \mathrm{mg} / \mathrm{mL}$, respectively, which were higher than that of the positive control kojic acid $\left(\mathrm{IC}_{50}=0.39 \pm 0.02 \mu \mathrm{g} / \mathrm{mL}\right)(p<0.05)$. It can be seen that the tyrosinase inhibitory activity of the $P$. utilis flower extract is the highest, followed by the leaf extract, and the stem extract had the lowest tyrosinase inhibitory activity. In addition, the inhibitory activity of three samples on tyrosinase was related to the total phenol and total flavonoid content, by comparing their corresponding total phenol and total flavonoid content. Our results showed that the ethanol extracts of flowers, leaves, and stems contained $p$-coumaric acid, which has been reported to have a good inhibitory effect on mushroom polyphenol oxidase, human tyrosinase, and cell melanin [32]. Therefore, the utilization of flower extracts with high content of coumaric acid may be helpful for fruit browning prevention and human skin whitening.

\section{Conclusions}

This study investigated the total phenols, total flavonoids, and polyphenol composition of ethanol extracts from flowers, leaves, and stems of $P$. utilis. Their antioxidant capacity and inhibitory activities against $\alpha$-glycosidase, lipase, and tyrosinase were also determined in vitro. The total phenols and total flavonoids were detected with the highest contents in flowers followed by leaves and stems. Among polyphenol compounds, rutin and isorhamnetin-3-O-rutinoside were the dominant compounds in flower and leaf ethanol extracts. As for the stem ethanol extract, rutin and isoschaftoside were the most abundant substances. Moreover, the antioxidant capacity and enzyme inhibitory capacity were detected with the highest value in the flower ethanol extract compared with others. The outcome of the work could provide support to the development and utilization of $P$. utilis in food and health products.

\section{Data Availability}

All data included in this study are available upon request by contacting the corresponding author.

\section{Conflicts of Interest}

There are no conflicts of interest regarding the publication of this article.

\section{Acknowledgments}

The present work was financially supported by the Science and Technology Project of Yunnan Province (202002AE320006-01-03 and 2019ZG001-4-1) and the Key Research and Development Program of Kunming City (No. 2019-1-N-25318000003141).

\section{References}

[1] D. Šamec, E. Karalija, I. Šola, V. Vujčić Bok, and B. SalopekSond, "The role of polyphenols in abiotic stress response: the influence of molecular structure," Pan, vol. 10, no. 1, p. 118, 2021.

[2] F. Shahidi and P. Ambigaipalan, "Phenolics and polyphenolics in foods, beverages and spices: antioxidant activity and health effects-a review," Journal of Functional Foods, vol. 18, pp. 820-897, 2015.

[3] V. Ponzo, I. Goitre, M. Fadda et al., "Dietary flavonoid intake and cardiovascular risk: a population-based cohort study," Journal of Translational Medicine, vol. 13, no. 1, pp. 218-313, 2015.

[4] Boua, B. Benson, L. Traoré, D. C. Ouattara, J.-A. Mamyrbekova-Békro, and Y.-A. Békro, "The changes in the contents of polyphenolic compounds of taro (Colocasia esculenta) upon domestic processing," World Journal of Pharmaceutical Research, vol. 10, pp. 102-111, 2021. 
[5] H. Cory, S. Passarelli, J. Szeto, M. Tamez, and J. Mattei, "The role of polyphenols in human health and food systems: a minireview," Frontiers in Nutrition, vol. 5, p. 87, 2018.

[6] M. Sobhani, M. H. Farzaei, S. Kiani, and R. Khodarahmi, "Immunomodulatory; anti-inflammatory/antioxidant effects of polyphenols: a comparative review on the parental compounds and their metabolites," Food Reviews International, vol. 37, no. 8, pp. 759-811, 2021.

[7] J. Xiang, M. Zhang, F. B. Apea-Bah, and T. Beta, "Hydroxycinnamic acid amide (HCAA) derivatives, flavonoid C-glycosides, phenolic acids and antioxidant properties of foxtail millet," Food Chemistry, vol. 295, pp. 214-223, 2019.

[8] B. A. Nikfarjam, F. Hajiali, M. Adineh et al., "Anti-inflammatory effects of quercetin and vitexin on activated human peripheral blood neutrophils: - the effects of quercetin and vitexin on human neutrophils," Journal of Pharmacopuncture, vol. 20, no. 2, pp. 127-131, 2017.

[9] R. Rituparna, A. Saha, P. Barua, and Atish, “Tea polyphenols egcg and tf restrict tongue and liver carcinogenesis simultaneously induced by $N$-nitrosodiethylamine in mice," Toxicology and Applied Pharmacology, vol. 300, pp. 34-46, 2016.

[10] X.-Q. Chen, T. Hu, Y. Han et al., "Preventive effects of catechins on cardiovascular disease," Molecules, vol. 21, no. 12, p. 1759, 2016.

[11] S. Huang, Y. Ma, C. Zhang, S. Cai, and M. Pang, "Bioaccessibility and antioxidant activity of phenolics in native and fermented Prinsepia utilis Royle seed during a simulated gastrointestinal digestion in vitro," Journal of Functional Foods, vol. 37, pp. 354-362, 2017.

[12] Q. Zhang, H.-X. Liu, H.-B. Tan, and S.-X. Qiu, "Novel highly oxygenated and B-Ring-Seco-Ent-Diterpene glucosides from the seeds of Prinsepia utilis," Tetrahedron, vol. 71, no. 50, pp. 9415-9419, 2015.

[13] Y. Q. Xu, Z. Yao, J. Y. Hu, J. Teng, Y. Takaishi, and H. Q. Duan, "Immunosuppressive terpenes from Prinsepia utilis," Journal of Asian Natural Products Research, vol. 9, no. 6-8, pp. 637-642, 2007.

[14] D. Sun, S. Huang, S. Cai, J. Cao, and P. Han, "Digestion property and synergistic effect on biological activity of purple rice (Oryza sativa L.) anthocyanins subjected to a simulated gastrointestinal digestion in vitro," Food Research International, vol. 78, pp. 114-123, 2015.

[15] J. Zhou, Y. Ma, Y. Jia, M. Pang, G. Cheng, and S. Cai, "Phenolic profiles, antioxidant activities and cytoprotective effects of different phenolic fractions from oil palm (elaeis guineensis jacq.) fruits treated by ultra-high pressure," Food Chemistry, vol. 288, pp. 68-77, 2019.

[16] X. Zhang, Y. Jia, Y. Ma, G. Cheng, and S. Cai, "Phenolic composition, antioxidant properties, and inhibition toward digestive enzymes with molecular docking analysis of different fractions from Prinsepia utilis Royle fruits," Molecules, vol. 2312 pages, 2018.

[17] S. Cai, O. Wang, M. Wang et al., "In vitro inhibitory effect on pancreatic lipase activity of subfractions from ethanol extracts of fermented oats (Avena sativa L.) and synergistic effect of three phenolic acids," Journal of Agricultural and Food Chemistry, vol. 60, no. 29, pp. 7245-7251, 2012.

[18] N. Baurin, E. Arnoult, T. Scior, Q. T. Do, and P. Bernard, "Preliminary screening of some tropical plants for anti-tyrosinase activity," Journal of Ethnopharmacology, vol. 82, no. 2-3, pp. 155-158, 2002.

[19] C. S. Sánchez, A. M. T. González, M. C. García-Parrilla, J. J. Q. Granados, H. López García De La Serrana, and M. C. López Martínez, "Different radical scavenging tests in virgin olive oil and their relation to the total phenol content," Analytica Chimica Acta, vol. 593, no. 1, pp. 103-107, 2007.

[20] H. Guo, S. Kandasamy, and M. H. Wang, "Total phenolic, flavonoid contents and free radical scavenging capacity of extracts from tubers of Stachys affinis," Biocatalysis and Agricultural Biotechnology, vol. 15, 2018.

[21] L. Zhang, Z.-c. Tu, H. Wang, Z.-f. Fu, Q.-h. Wen, and D. Fan, "Metabolic profiling of antioxidants constituents in artemisia selengensis leaves," Food Chemistry, vol. 186, pp. 123-132, 2015.

[22] P. Milica, D. Dragana, M. Saša et al., "Chemical characterization of fruit wine made from oblačinska sour cherry," The Scientific World Journal, vol. 2014, Article ID 454797, 9 pages, 2014.

[23] Z. Liu, Z. Ren, C. C. Chuang, E. Kandaswamy, T. Zhou, and L. Zuo, "Role of ROS and nutritional antioxidants in human diseases," Frontiers in physiology, vol. 9, p. 477, 2018.

[24] K. Cui, X. Luo, K. Xu, and M. R. Ven Murthy, "Role of oxidative stress in neurodegeneration: recent developments in assay methods for oxidative stress and nutraceutical antioxidants," Progress in Neuro-Psychopharmacology and Biological Psychiatry, vol. 28, no. 5, pp. 771-799, 2004.

[25] S. Cuevas and P. Pelegrín, "Pyroptosis and redox balance in kidney diseases," Antioxidants and Redox Signaling, vol. 35, no. 1, pp. 40-60, 2021.

[26] C. Zhang, Y. Ma, F. Gao, Y. Zhao, S. Cai, and M. Pang, "The free, esterified, and insoluble-bound phenolic profiles of rhus chinensis mill. Fruits and their pancreatic lipase inhibitory activities with molecular docking analysis," Journal of Functional Foods, vol. 40, pp. 729-735, 2018.

[27] A. C.-N. Leong, Y. Kinjo, M. Tako, H. Iwasaki, H. Oku, and H. Tamaki, "Flavonoid glycosides in the shoot system of Okinawa Taumu (Colocasia esculenta S.)," Food Chemistry, vol. 119, no. 2, pp. 630-635, 2010.

[28] R. Gao, Q. Yu, Y. Shen et al., "Production, bioactive properties, and potential applications of fish protein hydrolysates: developments and challenges," Trends in Food Science \& Technology, vol. 110, no. 1, pp. 687-699, 2021.

[29] M. J. Rahman, P. Ambigaipalan, and F. Shahidi, "Biological activities of camelina and sophia seeds phenolics: inhibition of LDL oxidation, DNA damage, and pancreatic lipase and $\alpha$-glucosidase activities," Journal of Food Science, vol. 83, no. 1-3, pp. 237-245, 2018.

[30] Y. Wu, Q. Zhou, X.-y. Chen, X. Li, Y. Wang, and J.-l. Zhang, "Comparison and screening of bioactive phenolic compounds in different blueberry cultivars: evaluation of anti-oxidation and $\alpha$-glucosidase inhibition effect," Food Research International, vol. 100, no. 1, pp. 312-324, 2017.

[31] S. Dorota, P. Anna, R. Małgorzata, and Z. Kucharska Alicja, "Inhibitory effect of black chokeberry fruit polyphenols on pancreatic lipase-searching for most active inhibitors," Journal of Functional Foods, vol. 49, pp. 196-204, 2018.

[32] C. B. Yong, "P-coumaric acid as an active ingredient in cosmetics: a review focusing on its antimelanogenic effects," Antioxidants, vol. 8, no. 8, p. 275, 2019. 